\title{
B-vitamins and their interaction with the MTHFR C677 T genotype as determinants of bone health in older adults from the TUDA Ageing cohort study
}

\author{
M. Clarke ${ }^{1,2}$, M. Ward ${ }^{1}$, L. Hoey ${ }^{1}$, J.J. Strain ${ }^{1}$, A. Molloy ${ }^{3}$, E. Laird ${ }^{3}$, M. Healy ${ }^{4}$, M.C. Casey ${ }^{5}$, \\ C. Cunningham ${ }^{5}$, M.T. McCann ${ }^{1}$ and H. McNulty ${ }^{1}$ \\ ${ }^{1}$ Northern Ireland Centre for Food and Health, University of Ulster, Coleraine, BT52 1SA, ${ }^{2}$ UCD Institute of Food and \\ Health, Belfield, University College Dublin, Dublin 4, Ireland, ${ }^{3}$ School of Medicine, Trinity College Dublin 2, Ireland, \\ ${ }^{4}$ The Department of Clinical Biochemistry, Central Pathology, St James's Hospital, Dublin, Ireland and ${ }^{5}$ The Mercers \\ Institute for Research on Ageing, St James's Hospital, Dublin, Ireland
}

\section{This abstract was presented as the Public Health Nutrition Theme highlight.}

Osteoporosis, characterised by reduced bone mineral density (BMD) and a high risk of fragility fracture, is increasingly prevalent in today's ageing society ${ }^{(1)}$. Large observational studies support a protective role for certain B-vitamins integral to 1-carbon metabolism (i.e. folate, vitamins B12, B6 and riboflavin) against osteoporosis ${ }^{(2)}$. A common polymorphism (C677 T) in the folate metabolising enzyme methylenetetrahydrofolate reductase (MTHFR) has also been linked to osteoporosis, with a heightened risk demonstrated in the presence of low B-vitamin status ${ }^{(2)}$. However, previous studies exploring the role of this polymorphism have tended to overlook relevant gene-nutrient interactions ${ }^{(2)}$. The aim of this investigation was to examine the interaction of relevant B-vitamin biomarkers with the MTHFR 677TT genotype as determinants of low BMD.

Older adults $(n 3,127)$ recruited to the Trinity Ulster Department of Agriculture (TUDA) Ageing cohort study, and with BMD measured by dual energy X-ray absorptiometry scans, were investigated. Low BMD was defined as a combination of osteopenia (T-Score; between -1 and $-2 \cdot 5 \mathrm{SD}$ ) and osteoporosis (T-Score; $-2.5 \mathrm{SD}$ or less).

Age $(p<0.001)$, physical inactivity $(p=0.015)$, and parathyroid hormone $(p<0.001)$ were associated with an increased risk of low BMD, while increasing body weight $(\mathrm{p}<0.001)$ reduced the risk. The MTHFR C677 T genotype was not found to be a significant predictor of low BMD in either males or females. Females with the MTHFR 677TT genotype in combination with low biomarker status of riboflavin or folate had a two-fold increased risk of low BMD compared to those with the MTHFR 677CC genotype and optimal B-vitamin status, after adjustment for covariates; these associations were not observed in men (Table).

Table. Predictors of low bone mineral density 'BMD' (osteoporosis and osteopenia combined)

\begin{tabular}{|c|c|c|c|}
\hline B-vitamin biomarker $(n 3,127)$ & OR & $95 \% \mathrm{CI}$ & $\mathrm{P}$ \\
\hline \multicolumn{4}{|l|}{ Males (n 1,056) } \\
\hline MTHFR 677TT genotype & $1 \cdot 047$ & $0.672-1.631$ & $0 \cdot 841$ \\
\hline Low riboflavin* $M T H F R$ 677TT genotype & 0.937 & $0 \cdot 476-1 \cdot 844$ & $0 \cdot 831$ \\
\hline Low folate* $M T H F R$ 677TT genotype & $1 \cdot 905$ & $0 \cdot 896-4 \cdot 049$ & 0.094 \\
\hline \multicolumn{4}{|l|}{ Females $(n$ 2,071) } \\
\hline MTHFR 677TT genotype & $1 \cdot 378$ & $0 \cdot 961-1 \cdot 976$ & $0 \cdot 081$ \\
\hline Low riboflavin* $M T H F R$ 677TT genotype & $2 \cdot 314$ & $1 \cdot 237-4 \cdot 329$ & $0 \cdot 009$ \\
\hline Low folate* MTHFR $677 \mathrm{TT}$ genotype & $2 \cdot 007$ & $1 \cdot 106-3 \cdot 642$ & $0 \cdot 022$ \\
\hline
\end{tabular}

Analysis by binary logistic regression, comparing lowest tertile of vitamin status to the other tertiles combined. Reference category; $M T H F R$ 677CC genotype and highest B-vitamin status. Riboflavin was measured using erythrocyte glutathionine reductase activation coefficient 'EGRac'. Folate was measured in red blood cells by microbiological assay.

These findings suggest that there are important gene-nutrient interactions within 1-carbon metabolism that appear to play an important role in maintaining bone health throughout life.

Acknowledgement of Funding: Food for Health Research Initiative of the Irish Department of Agriculture and Health Research Board, with co-funding from the Department for Employment and Learning Northern Ireland under its Cross-Border Research and Development Programme: "Strengthening the all-Island Research Base".

1. Strom O, Borgstrom F, Kanis JA et al. (2011) Arch Osteoporos 6, 59-155.

2. Dai \& Koh (2015) Nutrients, 7, 3322-3346. 